\title{
Topical issue on EChO - the Exoplanet Characterisation Observatory
}

\section{Foreword}

\author{
Giovanna Tinetti ${ }^{1} \cdot$ Pierre Drossart $^{2}$
}

Received: 12 November 2015 / Accepted: 12 November 2015 /Published online: 21 November 2015 (C) Springer Science+Business Media Dordrecht 2015

The concept of a mission devoted to atmospheric characterization of planets through transit spectroscopy was first considered in Europe in 2007, shortly after the DARWIN proposal submitted to the European Space Agency for the first Cosmic Vision call for Lclass missions was rejected because of scientific and technical immaturity. Following the decision, both ESA (EP-RAT panel - report in October 2010) and the Exoplanetary Community (Blue Dot Team - Barcelona conference in September 2009) started a discussion to define a scientific and technological roadmap for exoplanetary research. Both groups concluded that an intermediate step was needed, both scientifically and technically, before the characterisation of Earth-like planets could be tackled, and recommended a transit spectroscopy mission as a first step to atmospheric characterization.

EChO, the Exoplanet Characterisation Observatory, was proposed as a medium class mission candidate in 2010 by a consortium of institutes and universities from ESA member states, in response to the second call for medium class missions in the Cosmic Vision 2015-2025 programme. The mission was one of four selected in February 2011 for further study in a Phase 0/A assessment study (Tinetti et al., 2012, Experimental Astronomy, 34, 311-353). Soon after, an ESA study team, and a Science Study team comprising scientists from the proposing team and beyond, were assembled. An internal ESA pre-assessment study of EChO was undertaken in the Concurrent Design Facility at ESTEC that provided a baseline mission concept for subsequent study. The industrial and scientific payload studies started in early 2012 and concluded in September, 2013. The industrial studies performed by Astrium (Toulouse) and TAS-F (Cannes) included a review of the mission requirements, the technical design and a programmatic analysis of the mission. The study of the scientific payload including science ground segment elements and an end-to-end performance simulation

Giovanna Tinetti

g.tinetti@ucl.ac.uk

1 Department of Physics \& Astronomy, University College London, WC1E6BT London, UK

2 LESIA, Observatoire de Paris, Meudon, France 
of the complete system - was performed by the payload study team, i.e., a consortium of research institutes and universities from many ESA member states which has evolved since the selection with new members joining to participate in the instrument provision.

This special issue presents a summary of the very large body of work that has been undertaken on the EChO mission at scientific and technical levels over the 32-month period of the EChO pre-assessment and assessment phase. As such, it represents the contributions of a large number of parties (ESA, industry, institutes and universities), encompassing a very large number of people. At the end of the Phase-A study, EChO was considered a feasible mission within the ESA M3 cost-cap. Most importantly, no technical/scientific/programmatic showstoppers where identified (ESA-ECHO-RP-0001, http://sci.esa.int/echo/53519-echo-preliminary-requirementsreview-technical-report/\#). While EChO was not selected for the M3 launch opportunity, the scientific and the technical work accomplished during the Phase A are an important milestone towards the understanding of new worlds in our galaxy including the habitable ones - and how to progress towards that goal from a technical perspective. Out of the experience of $\mathrm{EChO}$, other similar space mission concepts were considered internationally. In particular, ARIEL - Atmospheric Remote-sensing Infrared Exoplanet Large-survey - has been selected in June 2015 as one of the three candidate missions for the M4 launch opportunity in ESA Cosmic Vision (launch in 2026).

ARIEL will focus on warm and hot planets, whereas EChO was also targeting temperate ones. Because of this choice, ARIEL wavelength range is narrower (1.95$7.8 \mu \mathrm{m}+$ photometric bands in the visible/Near Infrared on ARIEL as opposed to 0.5$16 \mu \mathrm{m}$ on EChO), allowing a much simpler payload: i.e., a simpler cooling system, one instrument module as opposed to three modules. Being launched after NASA TESS, and ESA Cheops and PLATO, a large number of bright transiting targets will be available for ARIEL. A combination of bright sources and increased throughput (because of the simpler payload), will enable the observation of hundreds of exoplanets, providing, for the first time, a statistical view of planets in our galaxy. Hot planets offer the unique opportunity to have access to the bulk and elemental composition, as there is no cold trap in their atmospheres for species such as $\mathrm{H}_{2} \mathrm{O}, \mathrm{NH}_{3}, \mathrm{CH}_{4}, \mathrm{SiO}, \mathrm{CO}_{2}, \mathrm{CO}$ and, depending on the temperature, metallic compounds e.g., $\mathrm{TiO}, \mathrm{VO}, \mathrm{CrH}$. The knowledge of hot planets is therefore imperative to understand the big-picture of planet formation and evolution before we focus on colder regimes. Additionally, a large fraction of the currently available/expected to be discovered planets will orbit very close to their star and therefore will be hot. Having a short annual period, these are the best targets for transit and eclipse spectroscopy measurements.

A long term scientific objective is to characterize the whole range of exoplanets, including, of course, potentially habitable ones. ARIEL would act as a pathfinder for future, even more ambitious campaigns. Over the EChO Phase-A study period the number of confirmed exoplanets increased from 500 to over 1000. The counting is currently 2000 and it is expected to grow to several tens of thousands in the next decade, providing an ever-more tantalising prospect of exploring the diversity of planets beyond our own Solar System.

Giovanna Tinetti and Pierre Drossart 